\title{
Output Feedback Stabilization by Reduced Order Finite Time Observers using a Trajectory Based Approach
}

Michael Malisoff
Frederic Mazenc

\author{
Saeed Ahmed
}

\begin{abstract}
We use finite time reduced order continuousdiscrete observers to solve an output feedback stabilization problem for a class of nonlinear time-varying systems whose outputs contain uncertainty. Unlike earlier works, our feedback is discontinuous, but it does not contain distributed control terms. Our trajectory based approach is based on a contractivity condition. We illustrate our control in the context of a tracking problem for nonholonomic systems in chained form.
\end{abstract}

Index Terms-Observer, stability, time-varying

\section{INTRODUCTION}

This paper continues our development and use of finite time observers that can cope with uncertain or intermittent output observations and nonlinearities, while also reducing the dimension of the required observers. While our work [10] provided full order finite time observers (whose dimensions equal the dimension of the original system) that allowed intermittent output observations, and our work in [11] provided reduced order observers that led to continuous output feedback controllers that have distributed terms (meaning, the control is implicitly defined by integrals that contain past control values), the present work provides an alternative to [11] in which the controls contain a mixture of continuous and discrete time dynamics (and therefore are called continuous-discrete) but do not contain any distributed control terms. This can help further reduce the computational burden by eliminating the need for distributed terms; see [2] and [13] for the relevance of distributed terms.

Our work is motivated by the importance of estimating values of solutions of systems, which produces challenges from the applied and theoretical viewpoints. Much of the observers literature is based on the Luenberger observer or other asymptotic observers (from [7] and [8]), which have been constructed for large families of nonlinear systems. On the other hand, there are applications that call for finite time state estimation, e.g., fault detection, where asymptotic observers may present the disadvantage that they only present a useful estimate after a transient period.

Finite time observers can be used to exactly construct the solutions in an arbitrarily short amount of time when there are no perturbations, and to quantify the effects of the

Malisoff is with the Department of Mathematics, Louisiana State University, Baton Rouge, LA 70803-4918, USA, malisoff@1su.edu.

Malisoff was supported by NSF Grant 1711299 .

Mazenc is with EPI DISCO INRIA-Saclay, Laboratoire des Signaux et Systèmes (L2S, UMR CNRS 8506), CNRS, CentraleSupélec, Université Paris-Sud, 3 rue Joliot Curie, 91192, Gif-sur-Yvette, France, frederic.mazenc@12s.centralesupelec.fr.

Ahmed is with Department of Electrical and Electronics Engineering, Graduate School of Engineering and Science, Bilkent University, Ankara 06800, Turkey, ahmed@ee.bilkent.edu.tr. perturbations on the estimation error. Finite time observers such as [6] and [16] use nonsmooth functions, but since they are based on homogeneity properties, they do not lend themselves to the design of smooth observers. Other finite time observers are computed using past values of the output or dynamic extensions; see [3] and [17] for linear systems, and see [12], [15], and [18] for analogs for nonlinear systems. These earlier finite time observers provide estimates for all of the state variables, which can produce redundancies because oftentimes, some state components are already available for measurement and therefore do not need to be estimated.

By adapting the results from [12] and [18], our work [11] constructed finite time reduced order observers for a family of nonlinear time-varying systems. We will use the main result of [11] as a key building block for our control design in this work, which we believe provides the first use of reduced order finite time observers for nonlinear timevarying systems that does not require distributed terms in the control. Time-varying systems are important because tracking problems can be recast as problems whose objectives are the stabilization of the zero equilibrium of a time-varying system (namely, the tracking error dynamics). As was the case for [4] and [1, Chapt. 4, Sec. 4.4.3], our work only provides estimates of the unmeasured variables, leading to a feedback control that can be computed using observer values and the perturbed measurements of the outputs. By reducing the order of the observer, this work provides more user friendly observers and feedbacks, where one computes the fundamental matrix for a system whose dimension is that of the unmeasured variable (instead of the higher dimension of the original system). This is valuable because of the difficulty of computing fundamental matrices for higher order systems.

After presenting our class of systems and assumptions and theorem in Section II, we provide key lemmas in Section III including a trajectory based result from [14]. We prove our theorem in Section IV, and we apply our method in the context of a nonholonomic dynamics in Section V. We close in Section VI with ideas for future research.

We use standard notation, in which the dimensions of our Euclidean spaces are arbitrary unless otherwise noted, and which will be simplified whenever no confusion would arise. We use $|\cdot|$ to denote the usual Euclidean norm and the induced matrix norm, $|\cdot|_{J}$ is the sup over any interval $J,|\cdot|_{\infty}$ is the usual essential supremum, and $I$ is the identity matrix in the dimension under consideration. Given a constant $\tau>0$ and a continuous function $\varphi: \mathbb{R} \rightarrow \mathbb{R}^{n}$ and values $t \geq 0$, we define $\varphi_{t}$ by $\varphi_{t}(m)=\varphi(t+m)$ for all $m \in[-\tau, 0]$. For each continuous function $\Omega: \mathbb{R} \rightarrow \mathbb{R}^{n \times n}$, let $\Phi_{\Omega}$ 
denote the function such that $\frac{\partial \Phi_{\Omega}}{\partial t}\left(t, t_{0}\right)=-\Phi_{\Omega}\left(t, t_{0}\right) \Omega(t)$ and $\Phi_{\Omega}\left(t_{0}, t_{0}\right)=I$ for all $t \in \mathbb{R}$ and $t_{0} \in \mathbb{R}$. Then $\mathcal{M}(t, s)=\Phi_{\Omega}^{-1}(t, s)$ is the fundamental solution associated to $\Omega$ for $\dot{x}=\Omega(t) x$; see [19, Lemma C.4.1]. We also use the standard definitions of input-to-state stability (or ISS) and $\mathcal{K} \mathcal{L}$ and $\mathcal{K}_{\infty}$ functions [5]. We say that a function $V:[0, \infty) \times \mathbb{R}^{n} \rightarrow[0, \infty)$ is proper and positive definite provided there are class $\mathcal{K}_{\infty}$ functions $\underline{\alpha}$ and $\bar{\alpha}$ such that the inequalities $\underline{\alpha}(|x|) \leq V(t, x) \leq \bar{\alpha}(|x|)$ hold for all $(t, x) \in[0, \infty) \times \mathbb{R}^{n}$. Finally, we say that a function $\rho$ : $[0, \infty) \times \mathbb{R}^{n} \rightarrow \mathbb{R}^{m}$ is globally Lipschitz with respect to its second variable uniformly in $t$ provided there exists a constant $\bar{\rho} \geq 0$ such that $|\rho(t, a)-\rho(t, b)| \leq \bar{\rho}|a-b|$ holds for all $t \geq 0$ and all $a$ and $b$ in $\mathbb{R}^{n}$. For simplicity, we assume that our initial times are always $t_{0}=0$ in our theorem.

\section{Statement of Problem And Assumptions}

We study systems of the form

$$
\left\{\begin{array}{c}
\dot{z}(t)=A_{1}(t) x_{r}(t)+B_{1}(t) u(t)+\rho_{1}(t, z(t))+f_{1}(t) \\
\dot{x}_{r}(t)=A_{2}(t) x_{r}(t)+B_{2}(t) u(t)+\rho_{2}(t, z(t))+f_{2}(t)
\end{array}\right.
$$

where $z$ is valued in $\mathbb{R}^{p}, x_{r}$ is valued in $\mathbb{R}^{n-p}$, the output is

$$
y(t)=z(t)+\epsilon(t),
$$

$p<n, \epsilon$ is an unknown bounded piecewise continuous function, $A_{i}$ and $B_{i}$ for $i=1,2$ are known piecewise continuous bounded matrix valued functions, $\rho=\left(\rho_{1}, \rho_{2}\right)$ is known and piecewise continuous with respect to $t, u$ is an input, and $f=\left(f_{1}, f_{2}\right)$ is an unknown locally bounded piecewise continuous function; see [11] for motivation for the systems (1). We next state our assumptions on (1); see [11, Remark 3] for ways to check Assumption 1.

Assumption 1: There exist a known constant $\tau>0$ and a known bounded function $L: \mathbb{R} \rightarrow \mathbb{R}^{(n-p) \times p}$ of class $C^{1}$ with a bounded first derivative such that for all $t \in \mathbb{R}$ and with the choice $H(t)=A_{2}(t)+L(t) A_{1}(t)$, the matrix

$$
\Lambda(t)=\Phi_{A_{2}}(t, t-\tau)-\Phi_{H}(t, t-\tau)
$$

is invertible. Also, $\Lambda^{-1}$ is a bounded function of $t$.

Assumption 2: There exist a function $u_{s}$ that is globally Lipschitz in its second variable uniformly in $t$, a $C^{1}$ proper and positive definite function $V$, positive constants $c_{1}$ and $c_{2}$, and a function $\gamma$ of class $\mathcal{K}_{\infty}$ such that the time derivative of $V$ along all solutions $\chi=\left(z, x_{r}\right)$ of

$$
\left\{\begin{aligned}
\dot{z}(t)= & A_{1}(t) x_{r}(t)+B_{1}(t) u(t) \\
& +\rho_{1}(t, z(t))+h_{1}(t) \\
\dot{x}_{r}(t)= & A_{2}(t) x_{r}(t)+B_{2}(t) u(t) \\
& +\rho_{2}(t, z(t))+h_{2}(t)
\end{aligned}\right.
$$

with the choice $u(t)=u_{s}\left(t, x_{r}(t)+\mu_{1}(t), z(t)+\mu_{2}(t)\right)$ satisfies $\dot{V}(t) \leq-c_{1} V(t, \chi(t))+\gamma(|(\mu, h)(t)|)$ for all choices of the locally bounded piecewise continuous functions $\mu=$ $\left(\mu_{1}, \mu_{2}\right): \mathbb{R} \rightarrow \mathbb{R}^{n}$ and $h=\left(h_{1}, h_{2}\right): \mathbb{R} \rightarrow \mathbb{R}^{n}$ and all $t \geq 0$, and such that its time derivative along all solutions $\chi$ of (1) with the choice $u(t)=0$ for all $t \geq 0$ satisfies $\dot{V}(t) \leq c_{2} V(t, \chi(t))+\gamma(|h(t)|)$.
Assumption 3: The function $\rho=\left(\rho_{1}, \rho_{2}\right)$ is globally Lipschitz in its second variable uniformly in $t$ and there is a function $\alpha \in \mathcal{K}_{\infty}$ such that $|\rho(t, a)| \leq \alpha(|a|)$ for all $a \in \mathbb{R}^{p}$ and $t \geq 0$.

In terms of the variable $\chi=\left(z, x_{r}\right)$ and the function

$$
\begin{aligned}
& \rho_{4}(t, z)=-\left[D(t) z+\rho_{3}(t, z)\right], \text { where } \\
& \rho_{3}(t, z)=L(t) \rho_{1}(t, z)+\rho_{2}(t, z) \text { and } \\
& D(t)=\dot{L}(t)-H(t) L(t)
\end{aligned}
$$

where $H$ and $L$ are from Assumption 1, we will prove:

Theorem 1: Let Assumptions 1-3 hold, and let $T>0$ be a constant such that

$$
\tau<\frac{T c_{1}}{c_{1}+c_{2}}
$$

and set $t_{i}=i T$ for each integer $i \geq 0$. Then we can construct functions $\bar{\beta} \in \mathcal{K} \mathcal{L}$ and $\bar{\gamma} \in \mathcal{K}_{\infty}$ such that the following ISS result is true: All solutions $\chi:[0, \infty) \rightarrow \mathbb{R}^{n}$ of (1), in closed loop with the control $u(t)=u_{\star}\left(t, \bar{x}_{r}(t), y(t)\right)$ where

$$
\begin{aligned}
& u_{\star}\left(t, \bar{x}_{r}(t), y(t)\right)= \\
& \begin{cases}u_{s}\left(t, \bar{x}_{r}(t), y(t)\right) & \text { if } t \in \cup_{i \geq 0}\left[t_{i}, t_{i+1}-\tau\right) \\
0 & \text { otherwise }\end{cases}
\end{aligned}
$$

and where $\bar{x}_{r}$ is the state of the continuous-discrete observer

$$
\begin{aligned}
\dot{\bar{x}}_{r}(t)= & A_{2}(t) \bar{x}_{r}(t)+B_{2}(t) u_{\star}\left(t, \bar{x}_{r}(t), y(t)\right) \\
& +\rho_{2}(t, y(t)) \text { when } t \in \cup_{i \geq 0}\left[t_{i}, t_{i+1}-\tau\right) \\
\bar{x}_{r}\left(t_{i}\right)= & \Lambda\left(t_{i}\right)^{-1} \int_{t_{i}-\tau}^{t_{i}}\left[\Phi_{A_{2}}\left(m, t_{i}-\tau\right) \rho_{2}(m, y(m))\right. \\
& \left.+\Phi_{H}\left(m, t_{i}-\tau\right) \rho_{4}(m, y(m))\right] \mathrm{d} m \\
& +\Lambda\left(t_{i}\right)^{-1}\left[\Phi_{H}\left(t_{i}, t_{i}-\tau\right) L\left(t_{i}\right) y\left(t_{i}\right)\right. \\
& \left.-L\left(t_{i}-\tau\right) y\left(t_{i}-\tau\right)\right] \text { for all } i \geq 1
\end{aligned}
$$

with $\bar{x}_{r}(0)=0$, satisfy $|\chi(t)| \leq \bar{\beta}(|\chi(0)|, t)+\bar{\gamma}\left(|(\epsilon, f)|_{[0, t]}\right)$ for all $t \geq 0$.

\section{KEY LEMMAS}

In this section, we provide two lemmas that we need to prove our theorem. The first lemma is from [11]. The second is a contractivity (or trajectory based) lemma from [14].

Lemma 1: Consider the system

$$
\left\{\begin{aligned}
\dot{z}(t) & =A_{1}(t) x_{r}(t)+\delta_{1}(t, z(t)) \\
\dot{x}_{r}(t) & =A_{2}(t) x_{r}(t)+\delta_{2}(t, z(t))
\end{aligned}\right.
$$

where $z$ is valued in $\mathbb{R}^{p}, x_{r}$ is valued in $\mathbb{R}^{n-p}$, the output is (2) where $\epsilon(t)$ is a piecewise continuous bounded function, $A_{i}$ for $i=1$ and 2 is piecewise continuous and bounded, and $\delta_{1}$ and $\delta_{2}$ are piecewise continuous with respect to $t$ and satisfy the requirements from Assumption 3 with the choice $\rho=\left(\delta_{1}, \delta_{2}\right)$. Let $\tau>0, H$, and $L$ be such that Assumption 1 holds, and choose $\delta_{3}(t, z)=L(t) \delta_{1}(t, z)+$ $\delta_{2}(t, z)$ and $\delta_{4}(t, z)=-\left[D(t) z+\delta_{3}(t, z)\right]$, where $D(t)=$ $\dot{L}(t)-H(t) L(t)$. Then for each solution of (9), we have

$$
\begin{aligned}
& x_{r}(t)=\Lambda(t)^{-1} \int_{t-\tau}^{t}\left[\Phi_{A_{2}}(m, t-\tau) \delta_{2}(m, y(m)-\epsilon(m))\right. \\
& \left.+\Phi_{H}(m, t-\tau) \delta_{4}(m, y(m)-\epsilon(m))\right] \mathrm{d} m \\
& +\Lambda(t)^{-1}\left[\Phi_{H}(t, t-\tau) L(t)(y(t)-\epsilon(t))\right. \\
& -L(t-\tau)(y(t-\tau)-\epsilon(t-\tau))]
\end{aligned}
$$

for all $t \geq \tau$, where $\Lambda$ is defined in (3). 
Lemma 2: Let $T_{*}>0$ be a constant. Let $w:\left[-T_{*}, \infty\right) \rightarrow$ $[0, \infty)$ be a piecewise continuous locally bounded function and $d:[0, \infty) \rightarrow[0, \infty)$ be piecewise continuous. Assume that there exists a constant $\lambda \in(0,1)$ such that

$$
w(t) \leq \lambda|w|_{\left[t-T_{*}, t\right]}+d(t)
$$

holds for all $t \geq 0$. Then the inequality

$$
w(t) \leq|w|_{\left[-T_{*}, 0\right]} e^{\frac{\ln (\lambda)}{T_{*}} t}+\frac{1}{1-\lambda}|d|_{[0, t]}
$$

holds for all $t \geq 0$.

\section{Summary OF Proof OF THEOREM 1}

Let us introduce the error variable $\tilde{x}_{r}(t)=x_{r}(t)-\bar{x}_{r}(t)$. Then for all integers $i \geq 0$, our formulas (1) and (8) give

$$
\begin{aligned}
\dot{\tilde{x}}_{r}(t)= & A_{2}(t) \tilde{x}_{r}(t)+\rho_{2}(t, z(t))+f_{2}(t) \\
& -\rho_{2}(t, z(t)+\epsilon(t)) \text { for all } t \in\left(t_{i}, t_{i+1}\right) \\
\tilde{x}_{r}\left(t_{i}\right)= & x_{r}\left(t_{i}\right)-\bar{x}_{r}\left(t_{i}\right) .
\end{aligned}
$$

Since $u_{\star}\left(t, \bar{x}_{r}(t), y(t)\right)=0$ for all $t \in \cup_{i \geq 1}\left[t_{i}-\tau, t_{i}\right)$,

$$
\begin{aligned}
& x_{r}\left(t_{i}\right)=\Lambda\left(t_{i}\right)^{-1} \int_{t_{i}-\tau}^{t_{i}}\left[\Phi_{A_{2}}\left(m, t_{i}-\tau\right) \rho_{2}(m, z(m))\right. \\
& \left.+\Phi_{H}\left(m, t_{i}-\tau\right) \rho_{4}(m, z(m))\right] \mathrm{d} m+\Delta\left(t_{i}\right) \\
& +\Lambda\left(t_{i}\right)^{-1}\left[\Phi_{H}\left(t_{i}, t_{i}-\tau\right) L\left(t_{i}\right) z\left(t_{i}\right)-L\left(t_{i}-\tau\right) z\left(t_{i}-\tau\right)\right]
\end{aligned}
$$

for all $i \geq 1$, where

$$
\begin{aligned}
& \Delta(t)=\Lambda(t)^{-1} \int_{t-\tau}^{t}\left[\Phi_{A_{2}}(m, t-\tau) f_{2}(m)\right. \\
& \left.-\Phi_{H}(m, t-\tau)\left(L(m) f_{1}(m)+f_{2}(m)\right)\right] \mathrm{d} m
\end{aligned}
$$

for all $t \geq \tau$; this follows by applying Lemma 1 .

Consequently, our formulas (8) give

$$
\begin{aligned}
& \tilde{x}_{r}\left(t_{i}\right)=x_{r}\left(t_{i}\right)-\bar{x}_{r}\left(t_{i}\right)= \\
& \Lambda\left(t_{i}\right)^{-1} \int_{t_{i}-\tau}^{t_{i}}\left[\Phi _ { A _ { 2 } } ( m , t _ { i } - \tau ) \left\{\rho_{2}(m, z(m))\right.\right. \\
& \left.\left.-\rho_{2}(m, y(m))\right\}\right] \mathrm{d} m \\
& +\Lambda\left(t_{i}\right)^{-1} \int_{t_{i}-\tau}^{t_{i}}\left[\Phi _ { H } ( m , t _ { i } - \tau ) \left\{\rho_{4}(m, z(m))\right.\right. \\
& \left.\left.-\rho_{4}(m, y(m))\right\}\right] \mathrm{d} m \\
& +\Lambda\left(t_{i}\right)^{-1}\left[\Phi_{H}\left(t_{i}, t_{i}-\tau\right) L\left(t_{i}\right)\left(z\left(t_{i}\right)-y\left(t_{i}\right)\right)\right. \\
& \left.-L\left(t_{i}-\tau\right)\left(z\left(t_{i}-\tau\right)-y\left(t_{i}-\tau\right)\right)\right]+\Delta\left(t_{i}\right)
\end{aligned}
$$

for all $i \geq 1$. From Assumptions 1 and 3 and the bound $\sup _{t_{i}-\tau<m<t_{i}}\left|\Phi_{A_{2}}\left(m, t_{i}-\tau\right)\right| \leq \tau e^{\tau\left|A_{2}\right|_{\infty}}$ (and an analogous bound for $H$, which follow from Gronwall's inequality), we can use (15) to find a constant $c_{4}>0$ such that

$$
\left|\tilde{x}_{r}\left(t_{i}\right)\right| \leq c_{4} \sup _{s \in\left[t_{i}-\tau, t_{i}\right]}|(\epsilon, f)(s)|
$$

for all $i \geq 1$, namely,

$$
\begin{aligned}
& c_{4}=\left|\Lambda^{-1}\right|_{\infty}\left\{e^{\tau\left|A_{2}\right|_{\infty}(\bar{\rho}+1) \tau+}\right. \\
& \tau e^{\left.\tau|H|_{\infty}\left[|D|_{\infty}+\left(|L|_{\infty}+1\right)(\bar{\rho}+1)\right]+\left(1+e^{\tau|H|_{\infty}}\right)|L|_{\infty}\right\}}
\end{aligned}
$$

where $\bar{\rho}$ is a global Lipschitz constant for $\rho=\left(\rho_{1}, \rho_{2}\right)$ satisfying Assumption 3's Lipschitzness requirement.

Moreover, by integrating the first equality in (12), we deduce that there is a constant $c_{5}>0$ such that

$$
\begin{aligned}
& \left|\tilde{x}_{r}(t)\right| \leq c_{5}\left|\tilde{x}_{r}\left(t_{i}\right)\right| \\
& +c_{5} \int_{t_{i}}^{t}\left|\rho_{2}(\ell, z(\ell))-\rho_{2}(\ell, z(\ell)+\epsilon(\ell))+f_{2}(\ell)\right| \mathrm{d} \ell
\end{aligned}
$$

for all $t \in\left(t_{i}, t_{i+1}\right)$ and $i \geq 0$, namely, $c_{5}=e^{\left|A_{2}\right|_{\infty} T}$. It follows from (16) and Assumption 3 that for all $t \in\left[t_{i}, t_{i+1}\right]$ and $i \geq 1$, we have

$$
\begin{aligned}
\left|\tilde{x}_{r}(t)\right| \leq & c_{5} c_{4} \sup _{s \in\left[t_{i}-\tau, t_{i}\right]}|(\epsilon, f)(s)| \\
& +c_{5}(\bar{\rho}+1) \int_{t_{i}}^{t}|(\epsilon, f)(\ell)| \mathrm{d} \ell \\
\leq & c_{6} \sup _{s \in\left[t_{i}-\tau, t\right]}|(\epsilon, f)(s)|
\end{aligned}
$$

where $c_{6}=c_{5}\left(c_{4}+(\bar{\rho}+1) T\right)$. Next observe that the closedloop system from the conclusion of the theorem is

$$
\left\{\begin{aligned}
\dot{z}(t)= & A_{1}(t) x_{r}(t)+B_{1}(t) u_{a}(t) \\
& +\rho_{1}(t, z(t))+f_{1}(t) \\
\dot{x}_{r}(t)= & A_{2}(t) x_{r}(t)+B_{2}(t) u_{a}(t) \\
& +\rho_{2}(t, z(t))+f_{2}(t)
\end{aligned}\right.
$$

where $u_{a}(t)=u_{\star}\left(t, x_{r}(t)-\tilde{x}_{r}(t), z(t)+\epsilon(t)\right)$. We deduce from Assumption 2 that, for all $t \in\left[t_{i}-\tau, t_{i}\right)$ and $i \geq 1$,

$$
\dot{V}(t) \leq c_{2} V(t, \chi(t))+\gamma\left(|f|_{[0, t]}\right)
$$

and, when $t \in\left[t_{i}, t_{i+1}-\tau\right)$ and $i \geq 1$, we have $\dot{V}(t) \leq$ $-c_{1} V(t, \chi(t))+\gamma\left(\left|\left(\tilde{x}_{r}, \epsilon, f\right)(t)\right|\right)$.

Therefore, when $t \in\left[t_{i+1}-\tau, t_{i+1}\right)$ and $i \geq 0$, we have (20), while when $t \in\left[t_{i}, t_{i+1}-\tau\right)$ and $i \geq 1$, we have

$$
\dot{V}(t) \leq-c_{1} V(t, \chi(t))+\gamma\left(\left(c_{6}+1\right)|(\epsilon, f)|_{[0, t]}\right),
$$

by (18). Combining the previous two cases gives

$$
\begin{aligned}
V(t, \chi(t)) \leq & e^{c_{2} \tau-(T-\tau) c_{1}} V(t-T, \chi(t-T)) \\
& +T^{\sharp} \gamma\left(\left(c_{6}+1\right)|(\epsilon, f)|_{[0, t]}\right)
\end{aligned}
$$

for all $t \geq 2 T$, for a suitable constant $T^{\sharp}$ that only depends on $c_{1}, c_{2}$, and $\tau$, and where (22) was obtained by separately considering the cases where $t \in\left[t_{i}, t_{i+1}-\tau\right)$ or $t \in\left[t_{i}-\tau, t_{i}\right)$ for some $i$. Next note that (6) gives $c_{2} \tau-(T-\tau) c_{1}<0$. We can then complete the proof using Lemma 2, as follows.

We set $w(t)=V(t+2 T, \chi(t+2 T))$ and $d(t)=$ $T^{\sharp} \gamma\left(\left(c_{6}+1\right)|(\epsilon, f)|_{[0, t+2 T]}\right)$ along any solution of (19), and $\lambda=e^{c_{2} \tau-(T-\tau) c_{1}}$. Then $\lambda \in(0,1)$, so (10) from Lemma 2 is satisfied with $T_{*}=2 T$. Choosing class $\mathcal{K}_{\infty}$ functions $\underline{\alpha}$ and $\bar{\alpha}$ such that $\underline{\alpha}(|\chi|) \leq V(t, \chi) \leq \bar{\alpha}(|\chi|)$ for all $t$ and $\chi$, it follows from conclusion (11) of Lemma 2 that

$$
\begin{aligned}
\underline{\alpha}(|\chi(t)|) \leq & e^{\ln (\lambda) \max \{0, t-2 T\} /(2 T)} \bar{\alpha}\left(|\chi|_{[0,2 T]}\right) \\
& +\frac{T^{\sharp}}{1-\lambda} \gamma\left(\left(c_{6}+1\right)|(\epsilon, f)|_{[0, t]}\right)
\end{aligned}
$$

for all $t \geq 2 T$. Hence, we can use the fact that $\underline{\alpha}^{-1}(a+b) \leq$ $\underline{\alpha}^{-1}(2 a)+\underline{\alpha}^{-1}(2 b)$ for all nonnegative values $a$ and $b$ to get

$$
\begin{aligned}
|\chi(t)| \leq & \underline{\alpha}^{-1}\left(2 e^{\ln (\lambda) \max \{0, t-2 T\} /(2 T)} \bar{\alpha}\left(|\chi|_{[0,2 T]}\right)\right) \\
& +\underline{\alpha}^{-1}\left(\frac{2 T^{\sharp}}{1-\lambda} \gamma\left(\left(c_{6}+1\right)|(\epsilon, f)|_{[0, t]}\right)\right)
\end{aligned}
$$

for all $t \geq 2 T$. We can also find a constant $\bar{G}>0$ such that

$$
|\chi(t)| \leq e^{\max \{0,2 T-t\}} \bar{G}|\chi(0)|+\bar{G}|(\epsilon, f)|_{[0, t]}
$$

along all solutions of (19) for all $t \in[0,2 T]$. The ISS estimate now follows by using (25) to upper bound $|\chi|_{[0,2 T]}$ in (24), then using $\bar{\alpha}(a+b) \leq \bar{\alpha}(2 a)+\bar{\alpha}(2 b)$ for suitable nonnegative $a$ and $b$ and the same property for $\underline{\alpha}^{-1}$, and then taking the maximum of the resulting right sides of (24) and (25). 


\section{Application to NONHOLONOMIC System}

\section{A. Tracking problem}

Consider this variant of a system from [9, p. 143]:

$$
\left\{\begin{array}{l}
\dot{\xi}_{4}=\xi_{3} v_{1} \\
\dot{\xi}_{3}=\xi_{2} v_{1} \\
\dot{\xi}_{2}=v_{2} \\
\dot{\xi}_{1}=v_{1}
\end{array}\right.
$$

with $\left(\xi_{1}, \xi_{2}, \xi_{3}, \xi_{4}\right)$ valued in $\mathbb{R}^{4}$ and the input $\left(v_{1}, v_{2}\right)$ valued in $\mathbb{R}^{2}$, which is a nonholonomic system in chained form. We assume that $\xi_{4}, \xi_{2}$ and $\xi_{1}$ are measured, but that $\xi_{3}$ is not measured. Therefore, we cannot integrate $\dot{\xi}_{3}=\xi_{2} v_{1}$ to obtain $\xi_{3}(t)$ because $\xi_{3}(0)$ is not known. Instead, we consider the problem of making (26) track the trajectory

$$
\left(\xi_{1 r}(t), \xi_{2 r}(t), \xi_{3 r}(t), \xi_{4 r}(t)\right)=\left(t+\frac{1}{2} \sin (t), 0,0,0\right) .
$$

While the preceding problem was studied in [11], this earlier work produced a feedback control containing distributed terms (meaning, the feedback control contained an integral containing past control values). Here, we use Theorem 1 to produce a feedback control that is free of distributed control terms.

We use the change of variables and the feedback

$$
x_{1}=\xi_{1}-\xi_{1 r}(t) \text { and } v_{1}\left(t, x_{1}\right)=-x_{1}+1+\frac{1}{2} \cos (t)
$$

which produce the $x_{1}$ subsystem $\dot{x}_{1}=-x_{1}$ and which therefore prompts us to consider the problem of uniformly globally asymptotically stabilizing the tracking dynamics

$$
\left\{\begin{array}{l}
\dot{\xi}_{4}=\xi_{3}\left[1+\frac{1}{2} \cos (t)\right] \\
\dot{\xi}_{3}=\xi_{2}\left[1+\frac{1}{2} \cos (t)\right] \\
\dot{\xi}_{2}=v_{2}
\end{array}\right.
$$

to 0 , by replacing $x_{1}$ by 0 in the $\left(\xi_{2}, \xi_{3}, \xi_{4}, x_{1}\right)$ dynamics. This motivates us to apply Theorem 1 to

$$
\left\{\begin{aligned}
\dot{z} & =x_{r}\left[1+\frac{1}{2} \cos (t)\right] \\
\dot{x}_{r} & =u\left[1+\frac{1}{2} \cos (t)\right]+f_{2}(t)
\end{aligned}\right.
$$

where $u$ is the input, which one would study in the context of applying backstepping to (29).

\section{B. Applying Theorem 1}

The system (30) has the form (1) with $n=2, p=1$, $A_{1}(t)=B_{2}(t)=1+\frac{1}{2} \cos (t)$ and with $\rho_{1}, \rho_{2}, A_{2}, B_{1}$, and $f_{1}$ all being the zero function. We next show how to satisfy the assumptions of Theorem 1 for the system (30) with $y=z=\xi_{4}$. We choose $L(t)=-\frac{1}{3}$ for all $t \in \mathbb{R}$, which gives $H(t)=A_{2}(t)+L(t) A_{1}(t)=-\frac{1}{3}\left(1+\frac{1}{2} \cos (t)\right)$ for all $t \in \mathbb{R}$. Then for any constant $\tau>0$, we have $\Phi_{A_{2}}\left(t, t_{0}\right)=1$,

$$
\begin{aligned}
& \Phi_{H}\left(t, t_{0}\right)=e^{\frac{1}{3}\left(t-t_{0}\right)+\frac{1}{6}\left(\sin (t)-\sin \left(t_{0}\right)\right)}, \text { and } \Lambda(t)= \\
& 1-\Phi_{H}(t, t-\tau)=1-e^{\frac{\tau}{3}+\frac{1}{6}[\sin (t)-\sin (t-\tau)]},
\end{aligned}
$$

where $\Lambda$ is defined in (3). Since $\frac{1}{3} \tau+\frac{1}{6}[\sin (t)-\sin (t-\tau)] \geq$ $\frac{\tau}{6}>0$ for all $t \in \mathbb{R}$, the choice (31) of $\Lambda$ satisfies Assumption 1 for any $\tau>0$. Assumption 3 is satisfied with $\rho=0$.
We now check that Assumption 2 is satisfied with $u_{s}(t, \chi)=-2\left(x_{r}+z\right)$ and $V(\chi)=z^{2}+\frac{1}{2} x_{r}^{2}+z x_{r}$, where $\chi=\left(z, x_{r}\right)$. The system which corresponds to (4) is

$$
\left\{\begin{aligned}
\dot{z}(t)= & {\left[1+\frac{1}{2} \cos (t)\right] x_{r}+h_{1}(t) } \\
\dot{x}_{r}(t)= & {\left[1+\frac{1}{2} \cos (t)\right]\left[-2\left(x_{r}+z\right)+\bar{\mu}(t)\right] } \\
& +h_{2}(t)
\end{aligned}\right.
$$

where $\bar{\mu}=-2\left(\mu_{1}+\mu_{2}\right)$. Along all solutions of (32),

$$
\begin{aligned}
& \dot{V}(t)=-2\left[1+\frac{1}{2} \cos (t)\right] V(\chi(t))+\left(2 z+x_{r}\right) h_{1}(t) \\
& +\left(x_{r}+z\right)\left[\bar{\mu}(t)\left(1+\frac{1}{2} \cos (t)\right)+h_{2}(t)\right] \\
& \leq-V(\chi(t))+\left\{\left(2 z+x_{r}\right) h_{1}(t)\right. \\
& \left.+\left(x_{r}+z\right)\left[\bar{\mu}(t)\left(1+\frac{1}{2} \cos (t)\right)+h_{2}(t)\right]\right\}
\end{aligned}
$$

holds for all $t \geq 0$. We can also use the inequality $z x_{r} \geq$ $-\frac{1}{3} x_{r}^{2}-\frac{3}{4} z^{2}$ to get $V(\chi) \geq \frac{1}{6} x_{r}^{2}+\frac{1}{4} z^{2}$ for all $\chi \in \mathbb{R}^{2}$. We easily deduce that $\dot{V}(t) \leq-\frac{1}{4} V(\chi)+\gamma(|(\mu, h)(t)|)$ holds along all solutions of the dynamics (32) for all $t \geq 0$, where $\gamma(s)=456 s^{2}$ by using the bounds $a b \leq \frac{1}{96} a^{2}+24 b^{2}$ and $a b \leq \frac{1}{48} a^{2}+12 b^{2}$ for suitable real numbers $a$ and $b$ to get

$$
\begin{aligned}
& \left(2 z+x_{r}\right) h_{1} \leq \frac{1}{96}\left(2 z+x_{r}\right)^{2}+24 h_{1}^{2} \\
& \leq \frac{1}{12}\left(z^{2}+x_{r}^{2}\right)+24 h_{1}^{2} \leq \frac{1}{2} V\left(z, x_{r}\right)+24 h_{1}^{2} \\
& \text { and }\left(x_{r}+z\right)\left[\bar{\mu}\left(1+\frac{1}{2} \cos (t)\right)+h_{2}(t)\right] \\
& \leq \frac{1}{48}\left(x_{r}+z\right)^{2}+12\left[\bar{\mu}\left(1+\frac{1}{2} \cos (t)\right)+h_{2}(t)\right]^{2} \\
& \leq \frac{1}{48}\left(x_{r}+z\right)^{2}+12\left(3\left|\mu_{1}+\mu_{2}\right|+\left|h_{2}(t)\right|\right)^{2} \\
& \leq \frac{1}{4} V\left(z, x_{r}\right)+12(36)|(\mu, h)(t)|^{2}
\end{aligned}
$$

respectively. Hence, we can choose $c_{1}=\frac{1}{4}$ in Assumption 2. To get a value for $c_{2}$, note that along all solutions of

$$
\left\{\begin{aligned}
\dot{z}(t) & =\left[1+\frac{1}{2} \cos (t)\right] x_{r}+h_{1}(t) \\
\dot{x}_{r}(t) & =h_{2}(t)
\end{aligned}\right.
$$

for all $t \geq 0$, we can use the triangle inequality to get

$$
\begin{aligned}
\dot{V}= & \left(2 z+x_{r}\right)\left(1+\frac{1}{2} \cos (t)\right) x_{r}+\left(2 z+x_{r}\right) h_{1} \\
& +\left(x_{r}+z\right) h_{2} \\
\leq & \frac{3}{2}\left(x_{r}^{2}+2\left|x_{r} z\right|\right)+\frac{1}{2}|h|^{2}+\frac{1}{2}\left(2 z+x_{r}\right)^{2} \\
& +\frac{1}{2}\left(x_{r}+z\right)^{2} \\
\leq & \frac{3}{2}\left(2 x_{r}^{2}+z^{2}\right)+5\left(x_{r}^{2}+z^{2}\right)+\frac{1}{2}|h|^{2} \\
\leq & 48\left(\frac{1}{6}\left(z^{2}+x_{r}^{2}\right)\right)+\frac{1}{2}|h|^{2} \leq 48 V\left(z, x_{r}\right)+\frac{1}{2}|h|^{2}
\end{aligned}
$$

which allows us to choose $c_{2}=48$ to satisfy Assumption 2 . Therefore, we can apply Theorem 1 using $D(t)=\dot{L}(t)-$ $H(t) L(t)=-\frac{1}{9}\left(1+\frac{1}{2} \cos (t)\right)$.

By Theorem 1, it follows that the $\left(\xi_{4}, \xi_{3}\right)$-subsystem of

$$
\left\{\begin{array}{l}
\dot{\xi}_{4}=\left(1+\frac{1}{2} \cos (t)\right) \xi_{3} \\
\dot{\xi}_{3}=\left(1+\frac{1}{2} \cos (t)\right)\left(u_{\star}\left(t, \bar{\xi}_{3}, \xi_{4}\right)+\omega\right) \\
\dot{\omega}=v_{2}+2\left(1+\frac{\cos (t)}{2}\right) \xi_{3}
\end{array}\right.
$$

satisfies ISS with respect to $\omega$, where $\omega=\xi_{2}+2 \xi_{4}$ and

$u_{\star}\left(t, \bar{\xi}_{3}(t), \xi_{4}(t)\right)=$

$\begin{cases}-2\left(\bar{\xi}_{3}(t)+\xi_{4}(t)\right) & \text { if } t \in \cup_{i \geq 0}\left[t_{i}, t_{i+1}-\tau\right) \\ 0 & \text { otherwise }\end{cases}$

and $t_{i}=i T$ for all integers $i \geq 0$ and $\bar{\xi}_{3}$ is the state of the 
continuous-discrete observer that is defined by

$$
\left\{\begin{array}{l}
\dot{\bar{\xi}}_{3}(t)=\left(1+\frac{\cos (t)}{2}\right) u_{\star}\left(t, \bar{\xi}_{3}(t), \xi_{4}(t)\right) \\
\text { for all } t \in \cup_{i \geq 0}\left[t_{i}, t_{i+1}-\tau\right), \text { and } \\
\bar{\xi}_{3}\left(t_{i}\right)= \\
-\Lambda\left(t_{i}\right)^{-1} \int_{t_{i}-\tau}^{t_{i}} \Phi_{H}\left(m, t_{i}-\tau\right) D(m) \xi_{4}(m) \mathrm{d} m \\
-\frac{1}{3} \Lambda\left(t_{i}\right)^{-1}\left[\Phi_{H}\left(t_{i}, t_{i}-\tau\right) \xi_{4}\left(t_{i}\right)-\xi_{4}\left(t_{i}-\tau\right)\right] \\
\text { for all } i \geq 1
\end{array}\right.
$$

with $\bar{\xi}_{3}(0)=0$. We now use the change of feedback $v_{2}=$ $-\omega(t)-2 \bar{\xi}_{3}(t)(1+0.5 \cos (t))$ to obtain

$$
\begin{aligned}
\dot{\xi}_{4}(t) & =\left(1+\frac{1}{2} \cos (t)\right) \xi_{3}(t) \\
\dot{\xi}_{3}(t) & =\left(1+\frac{1}{2} \cos (t)\right)\left(u_{\star}\left(t, \bar{\xi}_{3}(t), \xi_{4}(t)\right)+\omega(t)\right) \\
\dot{\omega}(t) & =-\omega(t)+(2+\cos (t))\left(\xi_{3}(t)-\bar{\xi}_{3}(t)\right) .
\end{aligned}
$$

Hence, the UGAS property for (38) follows under a suitable bound on the sample rate $T$ so that the $\omega$ subsystem of (38) is globally exponentially stable to 0 ; see the appendix below.

\section{CONCLUSIONS}

We advanced the state of the art for the design of observers for nonlinear systems, through the construction of reduced order finite time observers and corresponding output feedbacks that are free of distributed terms. Since our observers only required computing fundamental matrices for subsystems that have the dimensions of the unknown states, our method can reduce the computational burden relative to existing methods. We hope to combine Theorem 1 with the result of [10] to cover delays and disturbances in the input.

\section{ApPendiX: AnAlysis of $\omega$ SUbSystem of (38)}

To show the required exponential stability of the $\omega$ subsystem of (38), we will introduce a suitable condition on $T$. To this end, first note that when $A_{2}, \epsilon, f_{1}$, and $\rho_{2}$ are all zero, our formulas (12), (14), and (15) produce $\dot{\tilde{x}}_{r}(t)=f_{2}(t)$ and

$$
\Delta\left(t_{i}\right)=\Lambda^{-1}\left(t_{i}\right) \int_{t_{i}-\tau}^{t_{i}}\left(1-\Phi_{H}\left(m, t_{i}-\tau\right)\right) f_{2}(m) \mathrm{d} m
$$

and $\tilde{x}_{r}\left(t_{i}\right)=\Delta\left(t_{i}\right)$ for all $i \geq 1$, respectively. The preceding equalities imply that for all $i \geq 2$ and $t \in\left[t_{i}, t_{i+1}\right)$, we have

$$
\begin{aligned}
& \left|\tilde{x}_{r}(t)\right|=\left|\tilde{x}_{r}\left(t_{i}\right)+\int_{t_{i}}^{t} \dot{\tilde{x}}_{r}(\ell) \mathrm{d} \ell\right|=\mid \Lambda^{-1}\left(t_{i}\right) \int_{t_{i}-\tau}^{t_{i}}(1- \\
& \left.\Phi_{H}\left(m, t_{i}-\tau\right)\right) f_{2}(m) \mathrm{d} m+\int_{t_{i}}^{t} f_{2}(m) \mathrm{d} m \mid \\
& \leq 2 \int_{t-2 T}^{t}\left|f_{2}(m)\right| \mathrm{d} m,
\end{aligned}
$$

by the bound $\max _{t_{i}-\tau \leq m \leq t_{i}}\left|\Lambda^{-1}\left(t_{i}\right)\left(1-\Phi_{H}\left(m, t_{i}-\tau\right)\right)\right| \leq$ 1 (which follows from the monotonicity of $\Phi_{H}\left(m, t_{i}-\tau\right)$ as a function of $m$ ). Specializing the preceding analysis to our case where $x_{r}=\xi_{3}$ and $f_{2}=(1+0.5 \cos (t)) \omega$, we obtain

$$
\left|(2+\cos (t))\left(\xi_{3}(t)-\bar{\xi}_{3}(t)\right)\right| \leq 9 \int_{t-2 T}^{t}|\omega(m)| \mathrm{d} m .
$$

Hence, along all solutions of the $\omega$ subsystem of (38), we can use Jensen's inequality and then the triangle inequality to check that the time derivative of $V_{0}(\omega)=\frac{1}{2} \omega^{2}$ satisfies

$$
\begin{aligned}
& \dot{V}_{0}(\omega) \leq-\omega^{2}+9|\omega| \int_{t-2 T}^{t}|\omega(m)| \mathrm{d} m \\
& \leq-\frac{1}{2} \omega^{2}+81 T \int_{t-2 T}^{t} \omega^{2}(m) \mathrm{d} m
\end{aligned}
$$

for all $t \geq 2 T$. We now assume that $T<\frac{1}{18}$. This allows us to pick a constant $\epsilon_{0}>0$ close enough to zero such that $81 T^{2}\left(2+\epsilon_{0}\right)<\frac{1}{2}$. Then the time derivative of

$$
\begin{aligned}
& V_{0}^{\sharp}\left(\omega_{t}\right)=V_{0}(\omega(t)) \\
& +\frac{1}{2}\left(2+\epsilon_{0}\right) 81 T \int_{t-2 T}^{t} \int_{\ell}^{t} \omega^{2}(m) \mathrm{d} m \mathrm{~d} \ell
\end{aligned}
$$

along all solutions of the $\omega$ subsystem of (38) satisfies

$$
\begin{aligned}
\dot{V}_{0}^{\sharp}\left(\omega_{t}\right) \leq & -\left[\frac{1}{2}-\left(2+\epsilon_{0}\right)(9 T)^{2}\right] \omega^{2}(t) \\
& -\frac{81}{2} \epsilon_{0} T \int_{t-2 T}^{t} \omega^{2}(m) \mathrm{d} m
\end{aligned}
$$

for all $t \geq 2 T$, which implies that $V_{0}^{\sharp}$ and so also $\omega$ converge to zero exponentially (since the quantity in squared brackets in (A.3) is a positive constant), as desired.

\section{REFERENCES}

[1] F. Bonnans and P. Rouchon. Commande et Optimisation de Systèmes Dynamiques. Les Éditions de 1'Ecole Polytechnique, Palaiseau, France, 2005

[2] F. Cacace and A. Germani. Output feedback control of linear systems with input, state and output delays by chains of predictors. Automatica, 85:455-461, 2017.

[3] R. Engel and G. Kreisselmeier. A continuous time observer which converges in finite time. IEEE Transactions on Automatic Control, 47(7):1202-1204, 2002.

[4] F. Friedland. Reduced-order state obervers. In H. Unbehauen, editor, Control Systems, Robotics and Automation - Vol. VIII, pages 26-36. Eoless Publishers Co. Ltd., Oxford, United Kingdom, 2009.

[5] H. Khalil. Nonlinear Systems, Third Edition. Prentice Hall, Upper Saddle River, NJ, 2002.

[6] F. Lopez-Ramirez, A. Polyakov, D. Efimov, and W. Perruquetti. Finitetime and fixed-time observer design: Implicit Lyapunov function approach. Automatica, 87:52-60, 2018.

[7] D. Luenberger. Observers for multivariable systems. IEEE Transactions on Automatic Control, 11(2):190-197, 1966.

[8] D. Luenberger. Introduction to Dynamic Systems. John Wiley and Sons, New York, 1979

[9] M. Malisoff and F. Mazenc. Constructions of Strict Lyapunov Functions. Springer, New York, NY, 2009.

[10] F. Mazenc, S. Ahmed, and M. Malisoff. Finite time estimation through a continuous-discrete observer. International Journal of Robust and Nonlinear Control, 28(16):4831-4849, 2018.

[11] F. Mazenc, S. Ahmed, and M. Malisoff. Reduced order finite time observers for time-varying nonlinear systems. In Proceedings of the IEEE Conference on Decision and Control, pages 2182-2186, Miami Beach, FL, 2018.

[12] F. Mazenc, E. Fridman, and W. Djema. Estimation of solutions of observable nonlinear systems with disturbances. Systems and Control Letters, 79:47-58, 2015.

[13] F. Mazenc and M. Malisoff. Stabilization of nonlinear time-varying systems through a new prediction based approach. IEEE Transactions on Automatic Control, 62(6):2908-2915, 2017.

[14] F. Mazenc, M. Malisoff, and S-I. Niculescu. Stability and control design for time-varying systems with time-varying delays using a trajectory-based approach. SIAM Journal on Control and Optimization, 55(1):533-556, 2017.

[15] P. Menold, R. Findeisen, and F. Allgower. Finite time convergent observers for nonlinear systems. In Proceedings of the IEEE Conference on Decision and Control, pages 5673-5678, Maui, HI, 2003.

[16] W. Perruquetti, T. Floquet, and E. Moulay. Finite time observers: application to secure communication. IEEE Transactions on Automatic Control, 53(1):356-360, 2008.

[17] T. Raff and F. Allgower. An observer that converges in finite time due to measurement-based state updates. IFAC Proceedings Volumes, 41(2):2693-2695, 2008.

[18] F. Sauvage, M. Guay, and D. Dochain. Design of a nonlinear finite time converging observer for a class of nonlinear systems. Journal of Control Science and Engineering, 2007(36954), 2007.

[19] E. Sontag. Mathematical Control Theory, Second Edition. Springer, New York, 1998. 